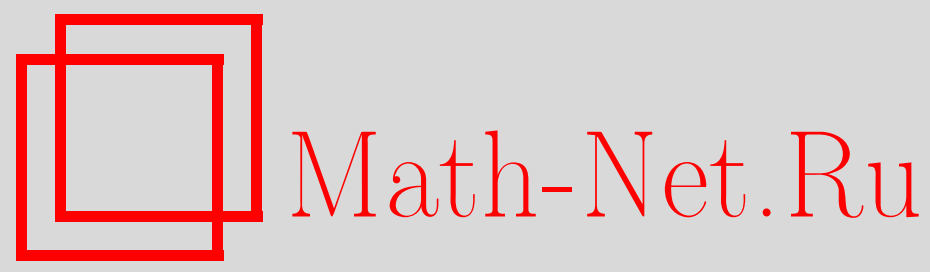

И. В. Неделько, Существование решений с внутренними слоями, выходящими на границу, Матем. заметки, 2005, том 77 , выпуск $1,80-92$

DOI: https://doi.org/10.4213/mzm2471

Использование Общероссийского математического портала Math-Net.Ru подразумевает, что вы прочитали и согласны с пользовательским соглашением http://www . mathnet.ru/rus/agreement

Параметры загрузки:

IP : 54.198 .64 .247

26 апреля 2023 г., $11: 32: 51$

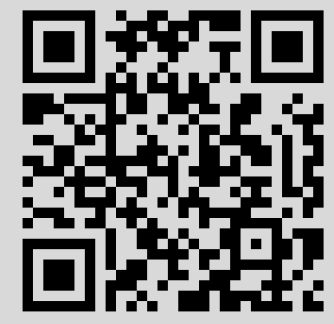




\section{СУЩЕСТВОВАНИЕ РЕШЕНИЙ С ВНУТРЕННИМИ СЛОЯМИ, ВЫХОДЯЩИМИ НА ГРАНИЦУ}

И.В. Неделько

Доказано существование решения с внутренним переходным слоем, выходящим на границу, в случае краевых условий Дирихле.

Библиография: 7 названий.

1. Введение. Рассмотрим задачу

$$
\begin{gathered}
\varepsilon^{2} \Delta u=f(u, x, \varepsilon), \quad x \in D \subset \mathbb{R}^{2}, \\
u(x, \varepsilon)=g(x), \quad x \in \partial D,
\end{gathered}
$$

где $\varepsilon>0$ - мальй параметр, $\Delta$ - оператор Лапласа, $x=\left(x_{1}, x_{2}\right), D$ - ограниченная односвязная область с достаточно гладкой границей $\partial D$.

Пусть выполнены следующие условия:

(А1) существует интервал $I$ и число $\varepsilon_{0}>0$ такие, что $f(u, x, \varepsilon)$ - достаточно гладкая функция в области $I \times \bar{D} \times\left[0, \varepsilon_{0}\right]$;

(А2) вырожденное уравнение $f(u, x, 0)=0$ имеет три корня $u=\varphi_{i}(x), i=0,1,2$, таких, что при $x \in \bar{D}$

$$
\begin{gathered}
\varphi_{1}(x)<\varphi_{0}(x)<\varphi_{2}(x), \quad\left[\varphi_{1}(x), \varphi_{2}(x)\right] \subset I, \\
f_{u}\left(\varphi_{i}(x), x, 0\right)>0, \quad i=1,2, \quad f_{u}\left(\varphi_{0}(x), x, 0\right)<0,
\end{gathered}
$$

и между $\varphi_{1}$ и $\varphi_{2}$ нет других корней кроме $\varphi_{0}$.

Введем функцию $J(x)=\int_{\varphi_{1}(x)}^{\varphi_{2}(x)} f(u, x, 0) d u$. Пусть

(А3) существует кривая $\Gamma \subset \bar{D}$, возможно имеющая общие точки с границей $\partial D$ области $D$, такая, что

$$
J(x)=0, \quad x \in \Gamma
$$

пусть, далее, кривая $\Gamma$ разделяет область $D$ на две подобласти $D^{(+)}$и $D^{(-)}$и пусть существуют гладкие замкнутые кривые $C_{1} \subset D^{(+)}$и $C_{2} \subset D^{(-)}$, ограничивающие некоторые односвязные области $D_{1}$ и $D_{2}$ соответственно такие, что

$$
J(x)>0, \quad x \in D^{(+)} \backslash D_{1}, \quad J(x)<0, \quad x \in D^{(-)} \backslash D_{2} .
$$


Отметим, что условие (А3) заведомо выполняется, если кривая $Г$, удовлетворяющая соотношению (3), разделяет область $D$ на две подобласти $D^{(+)}$и $D^{(-)}$такие, что $J(x)>0$ при $x \in D^{(+)}$и $J(x)<0$ при $x \in D^{(-)}$. В этом случае в качестве кривых $C_{1}$ и $C_{2}$ можно взять, например, окружности, целиком лежащие в $D^{(+)}$и $D^{(-)}$соответственно. Пусть

$(\mathrm{A} 4)$ функция $g(x)$ из граничного условия (2) достаточно гладкая и удовлетворяет неравенствам

$$
\varphi_{1}(x) \leqslant g(x) \leqslant \varphi_{2}(x), \quad x \in \partial D .
$$

Основной результат данной работы (теорема 3 в п. 4) состоит в том, что при условиях (A1)-(A4) для достаточно малых $\varepsilon$ доказано существование решения $u_{s}(x, \varepsilon)$ задачи $(1),(2)$, удовлетворяющего предельному соотношению

$$
\lim _{\varepsilon \rightarrow 0} u_{s}(x, \varepsilon)= \begin{cases}\varphi_{1}(x), & x \in D^{(+)}, \\ \varphi_{2}(x), & x \in D^{(-)} .\end{cases}
$$

Из (5) следует, что в окрестности кривой $Г$ из условия (А3) решение $u_{s}(x, \varepsilon)$ имеет внутренний переходньй слой, поэтому, следуя терминологии, принятой в [1], такое решение можно отнести к контрастным структурам типа ступеньки (КСТC).

Впервые существование КСТС в задаче (1), (2) доказано в работе [2], причем в [2] важную роль играет условие, что кривая, в окрестности которой решение имеет переходный слой (кривая перехода), является гладкой, замкнутой и не имеет общих точек с границей области $D$. В нашем случае условие (А3) позволяет кривой Г иметь общие точки с $\partial D$, и в этом случае внутренний переходньй слой решения $u_{s}(x, \varepsilon)$ выходит на границу $\partial D$ области $D$. Ситуация усложняется еще и тем, что указанное решение имеет пограничньй слой в окрестности $\partial D$, который накладывается на внутренний слой в окрестностях общих точек Г и $\partial D$. Из-за этого обстоятельства известные методы оказались не применимыми, и вопрос о существовании у задачи $(1),(2)$ решений с внутренними переходными слоями, выходяшими на границу области $D$, оставался открытым.

Отметим, что метод, развитьй в данной работе, применим и в том случае, когда вместо (2) поставлено краевое условие второго рода (подробнее см. замечание 1 в п. 4). Такая задача менее интересна, поскольку тогда у решения отсутствует пограничньй слой. Этот случай рассмотрен в работе [3] другим методом.

Опишем кратко содержание дальнейших разделов.

В п. 2 показано, что при достаточно малых $\varepsilon$ существует решение $u_{s}(x, \varepsilon)$ из $C^{2}(\bar{D})$ задачи $(1),(2)$, удовлетворяюшее предельному соотношению

$$
\lim _{\varepsilon \rightarrow 0} u_{s}(x, \varepsilon)= \begin{cases}\varphi_{1}(x), & x \in \bar{D}_{1}, \\ \varphi_{2}(x), & x \in \bar{D}_{2},\end{cases}
$$

где $D_{1}$ и $D_{2}$ - области из условия (А3). Соответствующие рассуждения основаны на построении (негладких) верхнего и нижнего решений задачи $(1),(2)$.

В п. 3 доказано, что решение $u_{s}(x, \varepsilon)$, удовлетворяющее $(6)$, удовлетворяет также соотношению (5), причем предельньй переход в (5) равномерен на замкнутых множествах из $D^{(+)}$и $D^{(-)}$. Можно сказать, что центральная идея доказательства состоит в построении барьеров параболической задачи, соответствующей задаче $(1),(2)$, для которой $u_{s}(x, \varepsilon)$ является стационарным решением.

В п. 4 сформулирован основной результат данной работы (теорема 3 ) и приведены замечания, касающиеся дальнейших утверждений, которые можно обосновать с помощью изложенного метода. 


\section{2. Доказательство существования решения.}

ТЕорема 1. Пусть выполнены условия (A1)-(A4). Тогда при достаточно малых $\varepsilon$ существует решение $u_{s}(x, \varepsilon)$ из $C^{2}(\bar{D})$ задачи (1), (2), причем $\forall \eta>0 \quad \exists \varepsilon^{\prime}>0$ такое, что при $\varepsilon \in\left(0, \varepsilon^{\prime}\right]$ это решение удовлетворяет неравенствам

$$
\begin{aligned}
u_{s}(x, \varepsilon)<\varphi_{1}(x)+\eta, & x \in \bar{D}_{1}, \\
u_{s}(x, \varepsilon)>\varphi_{2}(x)-\eta, & x \in \bar{D}_{2}, \\
\varphi_{1}(x)-\eta<u_{s}(x, \varepsilon)<\varphi_{2}(x)+\eta, & x \in \bar{D},
\end{aligned}
$$

где $D_{1}$ и $D_{2}-$ области из условия (A3).

ДокАЗАТЕЛЬСТво. Пусть $C_{\beta}$ и $C_{\alpha}$ - гладкие замкнутые кривые, ограничиваюшие односвязные области $D_{\beta}$ и $D_{\alpha}$ соответственно, такие, что

$$
D_{1} \subset D_{\beta} \subset D^{(+)}, \quad D_{2} \subset D_{\alpha} \subset D^{(-)},
$$

где $D^{(+)}, D^{(-)}$- области из условия (А 3$)$.

Для точек $M\left(x_{1}, x_{2}\right)$ из малой окрестности кривой $C_{\beta}$ введем локальные координаты $\left(r_{\beta}, \theta_{\beta}\right)$ (см. также [1]), где $\left|r_{\beta}\right|$ - расстояние от точки $M$ до кривой $C_{\beta}$ вдоль нормали к $C_{\beta}$, которая проходит через $M, \theta_{\beta}$ - координата той точки на $C_{\beta}$, из которой вьпущена указанная нормаль, $r_{\beta}=\left|r_{\beta}\right|$, если $M \in D_{\beta}, r_{\beta}=-\left|r_{\beta}\right|$, если $M \in D \backslash \bar{D}_{\beta}, r_{\beta}=0$, если $M \in C_{\beta}$.

Пусть $\varphi_{i}\left(r_{\beta}, \theta_{\beta}\right) \equiv \varphi_{i}\left(x\left(r_{\beta}, \theta_{\beta}\right)\right), i=1,2, f\left(u, r_{\beta}, \theta_{\beta}, \varepsilon\right) \equiv f\left(u, x\left(r_{\beta}, \theta_{\beta}\right), \varepsilon\right)$.

Аналогичным образом введем локальные координаты $\left(r_{\alpha}, \theta_{\alpha}\right)$ в окрестности кривой $C_{\alpha}$ и обозначения $\varphi_{i}\left(r_{\alpha}, \theta_{\alpha}\right), i=1,2, f\left(u, r_{\alpha}, \theta_{\alpha}, \varepsilon\right)$.

Построим функцию

$$
\begin{array}{lll}
\beta(x, \varepsilon)=\varphi_{1}(x)+\varepsilon^{1 / 2}+Q_{0 \beta}\left(\tau_{\beta}, \theta_{\beta}\right)+\varepsilon^{1 / 2} Q_{1 \beta}\left(\tau_{\beta}, \theta_{\beta}\right), & x \in \bar{D}_{\beta}, \\
\beta(x, \varepsilon)=\varphi_{2}(x)+\varepsilon^{1 / 2}, & x \in \overline{D \backslash D_{\beta}},
\end{array}
$$

где $\tau_{\beta}=r_{\beta} / \varepsilon$, причем при определении $Q_{0 \beta}$ и $Q_{1 \beta}$ будем пользоваться результатами работы [1].

Функция $Q_{0 \beta}\left(\tau_{\beta}, \theta_{\beta}\right)$ является решением задачи $\left(\theta_{\beta}\right.$ играет роль параметра)

$$
\begin{aligned}
\frac{\partial^{2} Q_{0 \beta}}{\partial \tau_{\beta}^{2}} & =f\left(\varphi_{1}\left(0, \theta_{\beta}\right)+Q_{0 \beta}, 0, \theta_{\beta}, 0\right), \quad \tau_{\beta}>0, \\
Q_{0 \beta}\left(0, \theta_{\beta}\right) & =\varphi_{2}\left(0, \theta_{\beta}\right)-\varphi_{1}\left(0, \theta_{\beta}\right), \quad Q_{0 \beta}\left(+\infty, \theta_{\beta}\right)=0 .
\end{aligned}
$$

В силу (10) из условия (А 3$)$ следует, что $J(x)>0$ при $x \in C_{\beta}$, поэтому $Q_{0 \beta}$ определяется однозначно, является положительной монотонно убьвающей функцией $\tau_{\beta}$ при $\tau_{\beta} \geqslant 0$ и имеет экспоненциальные оценки по переменной $\tau_{\beta}$ сверху и снизу, причем эти оценки равномерны по $\theta_{\beta}$, т.е. существуют постоянные $c_{i}>0$ и $m_{i}>0, i=1,2$, не зависящие от $\tau_{\beta}$ и $\theta_{\beta}$, такие, что

$$
\begin{array}{ll}
Q_{0 \beta}\left(\tau_{\beta}, \theta_{\beta}\right) \leqslant c_{1} \exp \left(-m_{1} \tau_{\beta}\right), & \tau_{\beta} \geqslant 0, \\
Q_{0 \beta}\left(\tau_{\beta}, \theta_{\beta}\right) \geqslant c_{2} \exp \left(-m_{2} \tau_{\beta}\right), & \tau_{\beta} \geqslant 0 .
\end{array}
$$


$\Phi$ ункцию $Q_{1 \beta}\left(\tau_{\beta}, \theta_{\beta}\right)$ определим как решение задачи

$$
\begin{gathered}
\frac{\partial^{2} Q_{1 \beta}}{\partial \tau_{\beta}^{2}}=f_{u}\left(\tau_{\beta}, \theta_{\beta}\right) Q_{1 \beta}+f_{1 \beta}\left(\tau_{\beta}, \theta_{\beta}\right), \quad \tau_{\beta}>0, \\
Q_{1 \beta}\left(0, \theta_{\beta}\right)=0, \quad Q_{1 \beta}\left(+\infty, \theta_{\beta}\right)=0,
\end{gathered}
$$

где

$$
\begin{aligned}
f_{u}\left(\tau_{\beta}, \theta_{\beta}\right) & \equiv f_{u}\left(\varphi_{1}\left(0, \theta_{\beta}\right)+Q_{0 \beta}, 0, \theta_{\beta}, 0\right), \\
f_{1 \beta}\left(\tau_{\beta}, \theta_{\beta}\right) & \equiv f_{u}\left(\tau_{\beta}, \theta_{\beta}\right)-f_{u}\left(\varphi_{1}\left(0, \theta_{\beta}\right), 0, \theta_{\beta}, 0\right) .
\end{aligned}
$$

$\Phi$ ункция $Q_{1 \beta}$ допускает явное представление [4] и имеет равномерную по $\theta_{\beta}$ экспоненциальную оценку сверху по переменной $\tau_{\beta}$ типа (13):

$$
\left|Q_{1 \beta}\left(\tau_{\beta}, \theta_{\beta}\right)\right| \leqslant c \exp \left(-m \tau_{\beta}\right), \quad \tau_{\beta} \geqslant 0 .
$$

Проведем стандартную процедуру домножения функций $Q_{0 \beta}$ и $Q_{1 \beta}$ на срезающие функции [1]. Теперь функция $\beta(x, \varepsilon)$ полностью построена.

Нетрудно доказать, что $\beta(x, \varepsilon)$ непрерывна в $\bar{D}$ и является достаточно гладкой в областях $\bar{D}_{\beta}$ и $\overline{D \backslash D_{\beta}}$, причем

$$
\begin{array}{ll}
\varepsilon^{2} \Delta \beta-f(\beta, x, \varepsilon)=-\varepsilon^{1 / 2} f_{u}\left(\varphi_{1}(x), x, 0\right)+O(\varepsilon)<0, & x \in \bar{D}_{\beta}, \\
\varepsilon^{2} \Delta \beta-f(\beta, x, \varepsilon)=-\varepsilon^{1 / 2} f_{u}\left(\varphi_{2}(x), x, 0\right)+O(\varepsilon)<0, & x \in \overline{D \backslash D_{\beta} .}
\end{array}
$$

Чтобы получить $(16),(17)$ в малой окрестности кривой $C_{\beta}$ следует перейти к локальным координатам $\left(r_{\beta}, \theta_{\beta}\right)$ (см. [1]).

Покажем, что при достаточно малых $\varepsilon$

$$
\left(\frac{\partial \beta}{\partial n_{\beta}}\right)_{\beta}^{(-)} \geqslant\left(\frac{\partial \beta}{\partial n_{\beta}}\right)_{\beta}^{(+)},
$$

где $\partial / \partial n_{\beta}-$ производная по направлению внутренней нормали к $C_{\beta}$, а символы $(\cdot)_{\beta}^{(-)}$и $(\cdot)_{\beta}^{(+)}$обозначают предельные значения на $C_{\beta}$ из областей $D \backslash D_{\beta}$ и $D_{\beta}$ соответственно. Действительно,

$$
\begin{aligned}
\varepsilon\left[\left(\frac{\partial \beta}{\partial n_{\beta}}\right)_{\beta}^{(-)}-\left(\frac{\partial \beta}{\partial n_{\beta}}\right)_{\beta}^{(+)}\right]= & \varepsilon\left[\varphi_{2 r}\left(0, \theta_{\beta}\right)-\varphi_{1 r}\left(0, \theta_{\beta}\right)\right] \\
& -\frac{\partial Q_{0 \beta}}{\partial \tau_{\beta}}\left(0, \theta_{\beta}\right)-\varepsilon^{1 / 2} \frac{\partial Q_{1 \beta}}{\partial \tau_{\beta}}\left(0, \theta_{\beta}\right) \\
= & -\frac{\partial Q_{0 \beta}}{\partial \tau_{\beta}}\left(0, \theta_{\beta}\right)+O\left(\varepsilon^{1 / 2}\right)
\end{aligned}
$$

С другой стороны, нетрудно получить [1] для $\frac{\partial Q_{0 \beta}}{\partial \tau_{\beta}}\left(0, \theta_{\beta}\right)$ соотношение

$$
\frac{\partial Q_{0 \beta}}{\partial \tau_{\beta}}\left(0, \theta_{\beta}\right)=-\left[2 \int_{\varphi_{1}\left(0, \theta_{\beta}\right)}^{\varphi_{2}\left(0, \theta_{\beta}\right)} f\left(u, 0, \theta_{\beta}, 0\right) d u\right]^{1 / 2}<0 .
$$


Из (20) и (19) при достаточно малых $\varepsilon$ следует (18).

Отметим также очевидное в силу (А4) неравенство

$$
\beta(x, \varepsilon)>g(x), \quad x \in \partial D .
$$

Аналогично функции $\beta(x, \varepsilon)$ построим функцию

$$
\begin{gathered}
\alpha(x, \varepsilon)=\varphi_{2}(x)-\varepsilon^{1 / 2}+Q_{0 \alpha}\left(\tau_{\alpha}, \theta_{\alpha}\right)-\varepsilon^{1 / 2} Q_{1 \alpha}\left(\tau_{\alpha}, \theta_{\alpha}\right), \quad x \in \bar{D}_{\alpha}, \\
\alpha(x, \varepsilon)=\varphi_{1}(x)-\varepsilon^{1 / 2}, \quad x \in \overline{D \backslash D_{\alpha}},
\end{gathered}
$$

где $\tau_{\alpha}=r_{\alpha} / \varepsilon$, а функции $Q_{0 \alpha}\left(\tau_{\alpha}, \theta_{\alpha}\right)$ и $Q_{1 \alpha}\left(\tau_{\alpha}, \theta_{\alpha}\right)$ определяются соответственно как решения задач

$$
\begin{aligned}
\frac{\partial^{2} Q_{0 \alpha}}{\partial \tau_{\alpha}^{2}} & =f\left(\varphi_{2}\left(0, \theta_{\alpha}\right)+Q_{0 \alpha}, 0, \theta_{\alpha}, 0\right), \quad \tau_{\alpha}>0, \\
Q_{0 \alpha}\left(0, \theta_{\alpha}\right) & =\varphi_{1}\left(0, \theta_{\alpha}\right)-\varphi_{2}\left(0, \theta_{\alpha}\right), \quad Q_{0 \alpha}\left(+\infty, \theta_{\alpha}\right)=0
\end{aligned}
$$

и

$$
\begin{gathered}
\frac{\partial^{2} Q_{1 \alpha}}{\partial \tau_{\alpha}^{2}}=f_{u}\left(\tau_{\alpha}, \theta_{\alpha}\right) Q_{1 \alpha}+f_{1 \alpha}\left(\tau_{\alpha}, \theta_{\alpha}\right), \quad \tau_{\alpha}>0, \\
Q_{1 \alpha}\left(0, \theta_{\alpha}\right)=0, \quad Q_{1 \alpha}\left(+\infty, \theta_{\alpha}\right)=0,
\end{gathered}
$$

где

$$
\begin{aligned}
f_{u}\left(\tau_{\alpha}, \theta_{\alpha}\right) & \equiv f_{u}\left(\varphi_{2}\left(0, \theta_{\alpha}\right)+Q_{0 \alpha}, 0, \theta_{\alpha}, 0\right) \\
f_{1 \alpha}\left(\tau_{\alpha}, \theta_{\alpha}\right) & \equiv f_{u}\left(\tau_{\alpha}, \theta_{\alpha}\right)-f_{u}\left(\varphi_{2}\left(0, \theta_{\alpha}\right), 0, \theta_{\alpha}, 0\right) .
\end{aligned}
$$

Так же как и в случае с $\beta(x, \varepsilon)$, нетрудно показать, что $\alpha(x, \varepsilon)$ непрерьвна в $\bar{D}$, но является, вообще говоря, негладкой на кривой $C_{\alpha}$, причем при достаточно малых $\varepsilon$

$$
\left(\frac{\partial \alpha}{\partial n_{\alpha}}\right)_{\alpha}^{(-)} \leqslant\left(\frac{\partial \alpha}{\partial n_{\alpha}}\right)_{\alpha}^{(+)}
$$

где $\partial / \partial n_{\alpha}-$ производная по направлению внутренней нормали к $C_{\alpha}$, а символы $(\cdot)_{\alpha}^{(-)}$и $(\cdot)_{\alpha}^{(+)}$обозначают предельные значения на $C_{\alpha}$ из областей $D \backslash D_{\alpha}$ и $D_{\alpha}$ соответственно. Кроме того, функция $\alpha(x, \varepsilon)$ построена таким образом, что вьполнены неравенства

$$
\begin{aligned}
\varepsilon^{2} \Delta \alpha-f(\alpha, x, \varepsilon)= & \varepsilon^{1 / 2} f_{u}\left(\varphi_{2}(x), x, 0\right)+O(\varepsilon)>0, \quad x \in \bar{D}_{\alpha}, \\
\varepsilon^{2} \Delta \alpha-f(\alpha, x, \varepsilon)= & \varepsilon^{1 / 2} f_{u}\left(\varphi_{1}(x), x, 0\right)+O(\varepsilon)>0, \quad x \in \overline{D \backslash D_{\alpha}}, \\
& \alpha(x, \varepsilon)<g(x), \quad x \in \partial D .
\end{aligned}
$$

Из (16)-(18), (21), (24)-(27) следует, что при достаточно малых $\varepsilon$ в соответствии с терминологией, принятой в [5], [6], функции $\beta(x, \varepsilon)$ и $\alpha(x, \varepsilon)$ являются соответственно (негладкими) верхним и нижним решениями задачи $(1),(2)$. Докажем, что при достаточно малых $\varepsilon$

$$
\beta(x, \varepsilon)>\alpha(x, \varepsilon), \quad x \in \bar{D} .
$$


Для этого покажем, что при достаточно малых $\varepsilon$ выполнено неравенство

$$
\beta(x, \varepsilon)>\varphi_{1}(x), \quad x \in \bar{D} \text {. }
$$

В области $\overline{D \backslash D_{\beta}}$ неравенство (29) очевидно (см. (11)).

Рассмотрим область $H_{1}=\left\{M\left(r_{\beta}, \theta_{\beta}\right) \in \bar{D}_{\beta}: 0 \leqslant r_{\beta} \leqslant r_{1}\right\}$, где $r_{1}=\kappa \varepsilon|\ln \varepsilon|, \kappa>0-$ число, которое выберем ниже. Так как $Q_{0}\left(\tau_{\beta}, \theta_{\beta}\right)$ имеет оценку $(14)$, то в области $H_{1}$, т.е. при $0 \leqslant \tau_{\beta} \leqslant \kappa|\ln \varepsilon|$, имеем

$$
Q_{0 \beta}\left(\tau_{\beta}, \theta_{\beta}\right) \geqslant c_{2} \varepsilon^{\kappa m_{2}}
$$

Выберем $\kappa>0$ столь малым, что $p=\kappa m_{2}<1 / 2$. Тогда получим, что при достаточно малых $\varepsilon$ в области $H_{1}$

$$
\beta(x, \varepsilon)-\varphi_{1}(x)=\varepsilon^{1 / 2}+Q_{0 \beta}\left(\tau_{\beta}, \theta_{\beta}\right)+\varepsilon^{1 / 2} Q_{1 \beta}\left(\tau_{\beta}, \theta_{\beta}\right) \geqslant \varepsilon^{1 / 2}+c_{2} \varepsilon^{p}-\varepsilon^{1 / 2} c>0,
$$

где $c>0-$ постоянная из оценки (15).

Для завершения доказательства неравенства (29) остается рассмотреть область $H_{2}=D_{\beta} \backslash H_{1}$. В этой области $\tau_{\beta}>\kappa|\ln \varepsilon|$, поэтому используя (15) и неотрицательность $Q_{0 \beta}$, при достаточно малых $\varepsilon$ получаем

$$
\beta(x, \varepsilon)-\varphi_{1}(x) \geqslant \varepsilon^{1 / 2}-\varepsilon^{1 / 2} c \varepsilon^{\kappa m}>0, \quad x \in H_{2},
$$

т.е. требуемое неравенство.

Аналогично (29) можно показать, что при достаточно малых $\varepsilon$

$$
\alpha(x, \varepsilon)<\varphi_{2}(x), \quad x \in \bar{D} .
$$

Теперь легко усмотреть, что в области $\bar{D}_{\beta}$ неравенство (28) непосредственное следствие $(29)$ и (22), а в области $\overline{D \backslash D_{\beta}}-$ следствие (30) и (11). Итак, (28) доказано.

Так как при достаточно малых $\varepsilon$ функции $\beta(x, \varepsilon)$ и $\alpha(x, \varepsilon)$ являются соответственно верхним и нижним решениями задачи $(1),(2)$ и вьполнено неравенство (28), то (согласно, например, теореме 1 из [5]; см. также теорему (1.1) в [7]) сушествует решение $u_{s}(x, \varepsilon)$ из $C^{2}(\bar{D})$ задачи $(1),(2)$ такое, что

$$
\alpha(x, \varepsilon)<u_{s}(x, \varepsilon)<\beta(x, \varepsilon), \quad x \in \bar{D} .
$$

Зафиксируем произвольное $\eta>0$.

Для завершения доказательства данной теоремы остается показать, что при достаточно малых $\varepsilon$ выполнены неравенства (7)-(9).

В силу экспоненциальных оценок сверху $Q$-фрункций

$$
\begin{array}{ll}
\beta(x, \varepsilon)=\varphi_{1}(x)+\varepsilon^{1 / 2}+O\left(\exp \left(-\frac{m_{3}}{\varepsilon}\right)\right), & x \in \bar{D}_{1}, \\
\alpha(x, \varepsilon)=\varphi_{2}(x)-\varepsilon^{1 / 2}+O\left(\exp \left(-\frac{m_{4}}{\varepsilon}\right)\right), & x \in \bar{D}_{2},
\end{array}
$$


где $m_{3}$ и $m_{4}-$ некоторые положительные постоянные, фиксированные при $\varepsilon \rightarrow 0$. Из $(31),(32)$ при достаточно малых $\varepsilon$ следуют (7) и (8).

Так как $Q_{0 \beta}\left(Q_{0 \alpha}\right)$ - монотонная функция по переменной $\tau_{\beta}\left(\tau_{\alpha}\right)$ и удовлетворяет условию (12) ((23)), можно утверждать, что при достаточно малых $\varepsilon$ в $\bar{D}_{\beta}\left(\bar{D}_{\alpha}\right)$ выполнено неравенство $\beta(x, \varepsilon)<\varphi_{2}(x)+\eta\left(\right.$ соответственно $\left.\alpha(x, \varepsilon)>\varphi_{1}(x)-\eta\right)$. Отсюда, а также из вида функций $\beta(x, \varepsilon)$ и $\alpha(x, \varepsilon)$ в $\overline{D \backslash D_{\beta}}$ и $\overline{D \backslash D_{\alpha}}$ соответственно следует, что при достаточно малых $\varepsilon$

$$
\beta(x, \varepsilon)<\varphi_{2}(x)+\eta, \quad \alpha(x, \varepsilon)>\varphi_{1}(x)-\eta, \quad x \in \bar{D} .
$$

Из последних неравенств и (31) получаем неравенства (9).

Теорема 1 доказана.

\section{3. Предельный переход при $\varepsilon \rightarrow 0$.}

ЛЕмма 1. Пусть выполнены условия (А1)-(А4) и пусть $v$ и щим иентром в некоторой точке $\bar{x}$ радиусов $R_{1}$ и $R_{2}$ соответственно, причем $R_{1}<R_{2}$, такие, что $\bar{V} \subset D^{(+)}\left(\bar{V} \subset D^{(-)}\right)$

$$
J(x)>0, \quad x \in \bar{V} \quad(J(x)<0, \quad x \in \bar{V})
$$

$u$, кроме того, $\forall \eta>0$ при достаточно малых $\varepsilon$ решение $u_{s}(x, \varepsilon)$ задачи (1), (2) из теоремы 1 удовлетворяет неравенству

$$
\begin{aligned}
u_{s}(x, \varepsilon)<\varphi_{1}(x)+\eta, & & x \in \bar{v} \\
\left(u_{s}(x, \varepsilon)>\varphi_{2}(x)-\eta,\right. & & x \in \bar{v}) .
\end{aligned}
$$

Тогда $\forall \eta^{\prime}>0$ при достаточно малых в выполняется неравенство

$$
\begin{aligned}
u_{s}(x, \varepsilon)<\varphi_{1}(x)+\eta^{\prime}, & x \in \bar{V} \\
\left(u_{s}(x, \varepsilon)>\varphi_{2}(x)-\eta^{\prime},\right. & x \in \bar{V}) .
\end{aligned}
$$

ДокАЗАТЕльство. Докажем первое утверждение данной леммы, т.е. утверждение, относящееся к неравенству (34). Второе утверждение (в скобках) доказьвается аналогично.

Пусть число $a \in\left(0, R_{1}\right)$ таково, что для круга $V_{1}$ радиуса $R_{2}+a$ с центром в точке $\bar{x}$ выполнены неравенства

$$
\bar{V}_{1} \subset D^{(+)}, \quad J(x)>0, \quad x \in \bar{V}_{1}
$$

Введем полярную систему координат $(\rho, \sigma)$ с полюсом в точке $\bar{x}$ и семейство окружностей $\Gamma_{\gamma}, \gamma \in[0,1]$, задав его в координатах $(\rho, \sigma)$ в виде

$$
\rho=R_{1}+\gamma\left(R_{2}+a-R_{1}\right) \equiv \rho_{\gamma}
$$

Очевидно, что

$$
\Gamma_{\gamma} \in \overline{V_{1}} \backslash v, \quad \gamma \in[0,1]
$$

окружность $\Gamma_{0}$ совпадает с границей круга $v$, а окружность $\Gamma_{1}-$ с границей круга $V_{1}$. 
Через $V_{\gamma}$ будем обозначать круг, ограниченньй $\Gamma_{\gamma}$. Пусть

$$
f(u, \rho, \sigma, \varepsilon) \equiv f(u, x(\rho, \sigma), \varepsilon), \quad \varphi_{i}(\rho, \sigma) \equiv \varphi_{i}(x(\rho, \sigma)), \quad i=1,2 .
$$

Построим функцию

$$
\hat{\beta}(x, \gamma, \mu)=\varphi_{1}(x)+\mu+Q_{0}\left(\tau_{\gamma}, \sigma, \gamma\right)+\mu Q_{1}\left(\tau_{\gamma}, \sigma, \gamma\right), \quad x \in \bar{V}_{\gamma}, \quad \gamma \in[0,1]
$$

где $\mu>0$ - число, которое уточним ниже,

$$
\tau_{\gamma}=\frac{\rho_{\gamma}-\rho}{\varepsilon}
$$

Функция $Q_{0}\left(\tau_{\gamma}, \sigma, \gamma\right)$ является решением задачи ( $\sigma$ и $\gamma$ играют роли параметров)

$$
\begin{gathered}
\frac{\partial^{2} Q_{0}}{\partial \tau_{\gamma}^{2}}=f\left(\varphi_{1}\left(\rho_{\gamma}, \sigma\right)+Q_{0}, \rho_{\gamma}, \sigma, 0\right), \quad \tau_{\gamma}>0 \\
Q_{0}(0, \sigma, \gamma)=\varphi_{2}\left(\rho_{\gamma}, \sigma\right)-\varphi_{1}\left(\rho_{\gamma}, \sigma\right), \quad Q_{0}(+\infty, \sigma, \gamma)=0 .
\end{gathered}
$$

Так как в силу $(35),(36) J(x)>0$ при $x \in \Gamma_{\gamma}, \gamma \in[0,1]$, то $Q_{0}$ определяется однозначно, является положительной монотонно убывающей функцией $\tau_{\gamma}$ при $\tau_{\gamma} \geqslant 0$ и имеет экспоненциальные оценки по переменной $\tau_{\gamma}$ сверху и снизу, причем эти оценки равномерны по $\gamma$ и $\sigma$.

Функцию $Q_{1}\left(\tau_{\gamma}, \sigma, \gamma\right)$ определим как решение задачи

$$
\begin{gathered}
\frac{\partial^{2} Q_{1}}{\partial \tau_{\gamma}^{2}}=f_{u}\left(\tau_{\gamma}, \sigma, \gamma\right) Q_{1}+f_{1}\left(\tau_{\gamma}, \sigma, \gamma\right), \quad \tau_{\gamma}>0 \\
Q_{1}(0, \sigma, \gamma)=0, \quad Q_{1}(+\infty, \sigma, \gamma)=0,
\end{gathered}
$$

где

$$
\begin{aligned}
f_{u}\left(\tau_{\gamma}, \sigma, \gamma\right) & \equiv f_{u}\left(\varphi_{1}\left(\rho_{\gamma}, \sigma\right)+Q_{0}, \rho_{\gamma}, \sigma, 0\right) \\
f_{1}\left(\tau_{\gamma}, \sigma, \gamma\right) & \equiv\left[f_{u}\left(\tau_{\gamma}, \sigma, \gamma\right)-f_{u}\left(\varphi_{1}\left(\rho_{\gamma}, \sigma\right), \rho_{\gamma}, \sigma, 0\right)\right]
\end{aligned}
$$

$\Phi$ ункция $Q_{1}$ допускает явное представление и имеет равномерную по $\gamma$ и $\sigma$ экспоненциальную оценку сверху по переменной $\tau_{\gamma}$.

Домножим функции $Q_{i}, i=1,2$, на срезающие функции так, чтобы

$$
\hat{\beta}(x, 1, \mu)=\varphi_{1}(x)+\mu, \quad x \in \bar{V},
$$

и получившиеся в результате этой процедуры функции снова обозначим через $Q_{i}, i=$ 1,2 .

По построению функция $\hat{\beta}(x, \gamma, \mu)$ удовлетворяет соотношению

$$
\varepsilon^{2} \Delta \hat{\beta}-f(\hat{\beta}, x, \varepsilon)=-\mu f_{u}\left(\varphi_{1}(x), x, 0\right)+O(\varepsilon)+O\left(\mu^{2}\right), \quad x \in \bar{V}_{\gamma}, \quad \gamma \in[0,1]
$$


(чтобы получить (38) в малой окрестности кривой $\Gamma_{\gamma}$ следует перейти к координатам $(\rho, \sigma)$, см. также [1]). Из (38) следует, что существует постоянная $c_{3}>0$, не зависящая от $\varepsilon, \mu$ и $\gamma$, такая, что для любого достаточно малого $\mu$ при достаточно малых $\varepsilon$ выполняется неравенство

$$
\varepsilon^{2} \Delta \hat{\beta}-f(\hat{\beta}, x, \varepsilon)<-c_{3} \mu, \quad x \in \bar{V}_{\gamma}, \quad \gamma \in[0,1]
$$

Используя положительность функции $Q_{0}$ и равномерные экспоненциальные оценки функций $Q_{i}$, нетрудно доказать, что для любого достаточно малого $\mu$ при достаточно малых $\varepsilon$ имеет место неравенство

$$
\hat{\beta}(x, \gamma, \mu)>\varphi_{1}(x)+\frac{\mu}{2}, \quad x \in \bar{V}_{\gamma}, \quad \gamma \in[0,1]
$$

Отметим также очевидное равенство

$$
\hat{\beta}(x, \gamma, \mu)=\varphi_{2}(x)+\mu, \quad x \in \Gamma_{\gamma}, \quad \gamma \in[0,1] .
$$

Из условия (33) и неравенств (9) следует, что для любого $\mu>0$ при достаточно малых $\varepsilon$ решение $u_{s}(x, \varepsilon)$ задачи $(1),(2)$ из теоремы 1 удовлетворяет неравенствам

$$
\begin{array}{ll}
u_{s}(x, \varepsilon)<\varphi_{1}(x)+\frac{\mu}{2}, & x \in \bar{v}, \\
u_{s}(x, \varepsilon)<\varphi_{2}(x)+\mu, & x \in \bar{D} .
\end{array}
$$

Кроме того, так как функция $u_{s}(x, \varepsilon)$ не зависит от времени, она удовлетворяет уравнению

$$
\varepsilon^{2} \Delta u_{s}-\frac{\partial u_{s}}{\partial t}=f\left(u_{s}, x, \varepsilon\right), \quad(x, t) \in D \times[0,+\infty) .
$$

Введем функцию

$$
w(x, t, \mu, \varepsilon)=\left[\hat{\beta}(x, \gamma(t), \mu)-u_{s}(x, \varepsilon)\right] \exp (-A t), \quad x \in \bar{V}_{\gamma(t)}, \quad t \in[0, T],
$$

где $\gamma(t)=t / T$, а величины $A$ и $T=T(\mu, \varepsilon)$ уточним ниже. Очевидно, что $0 \leqslant \gamma(t) \leqslant 1$ при $t \in[0, T], \gamma(0)=0, \gamma(T)=1$.

Используя (39) и (44), нетрудно получить неравенство

$$
\varepsilon^{2} \Delta w-w_{t}-B w<\left(-c_{3} \mu-\frac{\partial \hat{\beta}}{\partial \gamma} \cdot \frac{1}{T}\right) \exp (-A t), \quad x \in \bar{V}_{\gamma(t)}, \quad t \in[0, T]
$$

где

$$
B=B(x, t, \varepsilon, \mu)=A+\int_{0}^{1} f_{u}\left(u_{s}+\left(\hat{\beta}-u_{s}\right) p, x, \varepsilon\right) d p
$$

и, следовательно, $B>0$, если взять достаточно большое $A>0$.

Из вида $\hat{\beta}(x, \gamma, \mu)$ следует, что $\partial \hat{\beta} / \partial \gamma$ имеет оценку

$$
\left|\frac{\partial \hat{\beta}}{\partial \gamma}\right| \leqslant \frac{c_{4}}{\varepsilon}
$$


где постоянная $c_{4}>0$ независит от $\varepsilon, \gamma$, и $\mu$ (для ограниченного интервала изменения $\mu$ ). Поэтому, если взять $T=T(\mu, \varepsilon)$ такое, что

$$
T>\frac{c_{4}}{c_{3} \mu \varepsilon}
$$

то для любого достаточно малого $\mu$ при достаточно малых $\varepsilon$ будет вьполнено неравенство

$$
\varepsilon^{2} \Delta w-w_{t}-B w<0, \quad x \in \bar{V}_{\gamma(t)}, \quad t \in[0, T]
$$

Из (40) и (42) имеем

$$
w(x, 0, \mu, \varepsilon)>0, \quad x \in \bar{V}_{\gamma(0)}=\bar{v}
$$

а из (41) и (43) получаем

$$
w(x, t, \mu, \varepsilon)>0, \quad x \in \Gamma_{\gamma(t)}, \quad t \in[0, T] .
$$

Из (45)-(47) очевидным образом следует, что

$$
w(x, t, \mu, \varepsilon)>0, \quad x \in \bar{V}_{\gamma(t)}, \quad t \in[0, T]
$$

в частности, при $t=T$ имеем неравенство

$$
w(x, T, \mu, \varepsilon)>0, \quad x \in \bar{V}_{\gamma(T)}=\bar{V}_{1},
$$

откуда получаем

$$
u_{s}(x, \varepsilon)<\hat{\beta}(x, \gamma(T), \mu)=\hat{\beta}(x, 1, \mu), \quad x \in \bar{V}_{1} .
$$

Из (37) и (48) имеем

$$
u_{s}(x, \varepsilon)<\varphi_{1}(x)+\mu, \quad x \in \bar{V} .
$$

Теперь по заданному $\eta^{\prime}>0$ зафиксируем такое $\mu<\eta^{\prime}$, чтобы при достаточно малых $\varepsilon$ выполнялось неравенство (49). Так как $\mu<\eta^{\prime}$, наряду с (49) выполняется неравенство (34).

Лемма 1 доказана.

ТЕОрема 2. Пусть выполнены условия (A1)-(А4). Тогда $\forall \eta>0 u \forall$ замкнутого множества $\Omega^{(+)} \subset D^{(+)}\left(\right.$сответственно $\left.\Omega^{(-)} \subset D^{(-)}\right)$при достаточно малых $\varepsilon$ для решения $u_{s}(x, \varepsilon)$ задачи (1), (2) из теоремы 1 справедливы неравенства

$$
\begin{aligned}
& \varphi_{1}(x)-\eta<u_{s}(x, \varepsilon)<\varphi_{1}(x)+\eta, \quad x \in \Omega^{(+)} \\
& \left(\varphi_{2}(x)-\eta<u_{s}(x, \varepsilon)<\varphi_{2}(x)+\eta, \quad x \in \Omega^{(-)}\right) \text {. }
\end{aligned}
$$


ДоказАтЕльство. Докажем утверждение, относящееся к неравенству (50). Второе утверждение (в скобках) доказьвается аналогично.

Зафиксируем произвольные $\eta>0$ и замкнутое множество $\Omega^{(+)} \subset D^{(+)}$.

Справедливость неравенства $u_{s}(x, \varepsilon)>\varphi_{1}(x)-\eta$ при $x \in \Omega^{(+)}$следует из теоремы 1 (см. (9)).

Остается доказать, что при достаточно малых $\varepsilon$ выполнено неравенство

$$
u_{s}(x, \varepsilon)<\varphi_{1}(x)+\eta, \quad x \in \Omega^{(+)} .
$$

Воспользуемся тем, что $\forall r>0 \exists N>0$ и точки $x_{1}, \ldots, x_{N} \in D^{(+)}$такие, что

$$
\Omega^{(+)} \subset \bigcup_{i=1}^{N} E_{x_{i}}^{r},
$$

где $E_{x_{i}}^{r}-$ круги радиусов $r$ с центрами в точках $x_{i}$.

Обозначим через $D_{1}^{\prime}$ ту подобласть области $D_{1}$ (см. (А 3$)$ ), которая получается из $D_{1}$ после удаления точек, принадлежащих $3 r$-окрестности кривой $C_{1}$. Будем считать $r$ настолько малым, что: $D_{1}^{\prime} \neq \varnothing$, граница $\partial D_{1}^{\prime}$ области $D_{1}^{\prime}$ - гладкая замкнутая кривая без самопересечений,

$$
J(x)>0, \quad x \in D^{(+)} \backslash \bar{D}_{1}^{\prime},
$$

и $\bigcup_{i=1}^{N} \bar{E}_{x_{i}}^{r} \subset D^{(+)}$.

Зафиксируем произвольное натуральное $k \in 1, \ldots, N$ и покажем, что при достаточно малых $\varepsilon$

$$
u_{s}(x, \varepsilon)<\varphi_{1}(x)+\eta, \quad x \in \bar{E}_{x_{k}}^{r} .
$$

Если $\bar{D}_{1}^{\prime} \cap \bar{E}_{x_{k}}^{r} \neq \varnothing$, то $\bar{E}_{x_{k}}^{r} \subset D_{1}$ и (54) следует из теоремы 1.

Пусть $\bar{D}_{1}^{\prime} \cap \bar{E}_{x_{k}}^{r}=\varnothing$.

В этом случае возьмем произвольную точку $P \subset D_{1} \backslash \bar{D}_{1}^{\prime}$ и соединим ее некоторой кривой $L$ (например ломаной), лежащей в области $D^{(+)} \backslash \bar{D}_{1}^{\prime}$ с точкой $x_{k}$. Пусть число $d>0$ таково, что $2 d$-окрестность кривой $L$ целиком принадлежит области $D^{(+)} \backslash \bar{D}_{1}^{\prime}$. Отметим на кривой $L$ точки $y_{1}, \ldots, y_{n}, n \geqslant 2$, так, что $y_{1}=P, y_{n}=x_{k}$ и расстояние между $y_{i}$ и $y_{i+1}$ при $i=1, \ldots, n-1$ не превосходит $d / 2$.

Пусть $V_{1}, \ldots, V_{n-1}-$ круги радиуса $d$ с центрами в точках $y_{1}, \ldots, y_{n-1}$. Через $V_{n}$ обозначим круг $E_{x_{k}}^{r}$. Введем круги $v_{1}, \ldots, v_{n}$ с центрами в точках $y_{1}, \ldots, y_{n}$ и радиусами, меньшими, чем $d$ и $r$, так, что

$$
\begin{aligned}
v_{1} & \subset \bar{D}_{1}, \\
\bar{v}_{i+1} & \subset V_{i}, \quad i=1, \ldots, n-1 .
\end{aligned}
$$

Заметим, что в рассматриваемом случае $\bar{V}_{n}=\bar{E}_{x_{k}}^{r} \subset D^{(+)} \backslash \bar{D}_{1}^{\prime}$. С другой стороны, при $i=1, \ldots, n-1 \bar{V}_{i} \subset D^{(+)} \backslash \bar{D}_{1}^{\prime}$ в силу выбора $d$. Поэтому на основании (53) можно заключить, что при $i=1, \ldots, n$

$$
J(x)>0, \quad x \in \bar{V}_{i} .
$$


Пусть при некотором $j \in 1, \ldots, n-1 \forall \nu>0$ при достаточно малых $\varepsilon$ вьполнено неравенство

$$
u_{s}(x, \varepsilon)<\varphi_{1}(x)+\nu, \quad x \in \bar{V}_{j}
$$

а значит, в силу (56)

$$
u_{s}(x, \varepsilon)<\varphi_{1}(x)+\nu, \quad x \in \bar{v}_{j+1} .
$$

Тогда, учитывая (57), на основании леммы 1 можно заключить, что $\forall \nu>0$ при достаточно малых $\varepsilon$

$$
u_{s}(x, \varepsilon)<\varphi_{1}(x)+\nu, \quad x \in \bar{V}_{j+1} .
$$

Поэтому, чтобы обосновать (54), т.е. (58) с $\nu=\eta$ и $j=n$, достаточно показать, что $\forall \nu>0$ при достаточно малых $\varepsilon$ выполнено неравенство (58) для $j=1$, или, учитьвая лемму 1 - неравенство (59) для $j=0$. Справедливость неравенства (59) для $j=0$, в свою очередь, следует из теоремы 1 в силу (55).

Итак, при достаточно малых $\varepsilon$ вьполнено неравенство (54).

Так как число $N$ кругов $E_{x_{i}}^{r}$ конечно, а число $k \in 1, \ldots, N$ в (54) произвольно, на основании (54) можно заключить, что при достаточно малых $\varepsilon$

$$
u_{s}(x, \varepsilon)<\varphi_{1}(x)+\eta, \quad x \in \bigcup_{i=1}^{N} \bar{E}_{x_{i}}^{r}
$$

Из (60), (52) следует (51).

Теорема 2 доказана.

4. Основной результат. Сформулируем основной результат данной работы, непосредственно вытекающий из теоремы 2.

Теорема 3. Пусть выполнены условия (A1)-(A4). Тогда при достаточно малых $\varepsilon$ существует решение $u_{s}(x, \varepsilon)$ из $C^{2}(\bar{D})$ задачи $(1),(2)$, удовлетворяющее соотношению (5), причем предельный переход в (5) равномерен на замкнутых множсествах из $D^{(+)}$и $D^{(-)}$.

ЗАмЕчаниЕ 1 . Теорема 3 остается в силе, если краевое условие (2) первого рода заменить на краевое условие второго рода

$$
\left.\frac{\partial u}{\partial n}\right|_{\partial D}=g(x)
$$

где $\partial / \partial n$ - производная по направлению внешней нормали к $\partial D$. В этом случае неравенство (4) в условии (A4) не требуется, а изменится только доказательство теоремы 1: при построении $\beta(x, \varepsilon)$ и $\alpha(x, \varepsilon)$ (см. $(11),(22))$ нужно добавить погранслойные функции порядка $\varepsilon$, существенные только вблизи $\partial D$ и обеспечивающие вьполнение неравенств

$$
\left.\frac{\partial \beta}{\partial n}\right|_{\partial D} \geqslant g(x),\left.\quad \frac{\partial \alpha}{\partial n}\right|_{\partial D} \leqslant g(x) .
$$


ЗАмЕчАниЕ 2. Теорема 3 остается в силе, если вместо (А3) выполнено следующее требование:

$\left(\mathrm{A} 3^{\prime}\right)$ существует подобласть $D^{\prime}$ области $D$, в которой выполнено условие $(\mathrm{A} 3)$ с $D^{\prime}$ вместо $D$.

При этом вопрос о поведении решения, удовлетворяющего соотношению (5), в области $D \backslash D^{\prime}$ остается открытым.

ЗАмЕчАнИЕ 3. Если имеется несколько кривых $Г$, удовлетворяющих требованию $\left(\mathrm{A} 3^{\prime}\right)$ для различных подобластей $D^{\prime}$, то метод, представленньй в работе, позволяет установить существование решения задачи (1), (2), имеющего несколько переходных слоев.

\section{СПИСОК ЦИТИРОВАННОЙ ЛИТЕРАТУРЫ}

[1] Васильева А. Б., Бутузов В.Ф., Нефедов Н. Н. Контрастные структуры в сингулярно возмущенных задачах // Фундамент. и прикл. матем. 1998. Т. 4. № 3. С. 799-851.

[2] Файф П., Гринли В. Внутренние переходные слои для эллиптических краевых задач с малым параметром // УМН. 1974. Т. 29. № 4. С. 103-131.

[3] del Pino M. A. Layers with nonsmooth interface in a semilinear elliptic problem // Comm. Partial Differential Equations. 1992. V. 17. № 9. P. 1695-1708.

[4] Fife P. C. Semilinear elliptic boundary value problems with small parameters // Arch. Rat. Mech. Anal. 1973. V. 52. № 4. P. 205-232.

[5] Бутузов В. Ф., Неделько И. В. Асимптотическая устойчивость решений сингулярно возмущенных задач с пограничными и внутренними слоями // Дифференц. уравнения. 2000. Т. 36. №2. C. 198-208.

[6] Нефедов Н.Н. Метод дифференциальных неравенств для некоторых классов нелинейных сингулярно возмущенных задач с внутренними слоями // Дифференц. уравнения. 1995. Т. 31. №7. C. 1132-1139.

[7] Неделько И. В. Асимптотическая устойчивость и локальная единственность решений двумерных сингулярно возмущенных задач с пограничными и внутренними слоями. Дисс. ... к.ф.-м.н. М.: МГУ, 1999.

Московский государственный университет им. М.В. Ломоносова

E-mail: butuzov@mt384.phys.msu.su

Поступило

21.05.2001

Исправленный вариант

26.03.2004 